\title{
LOW CONCENTRATION EOSIN STAINING FOR HIGH CHEMICAL CONTRAST NONLINEAR MICROSCOPE MOSAIC IMAGING OF SKIN ALTERATIONS IN PSEUDOXANTHOMA ELASTICUM
}

\author{
Luca Fésűs ${ }^{1,2}$, Ludovic Martin, ${ }^{3}$ Norbert Wikonkál, ${ }^{1,2}$ Márta Medvecz ${ }^{2}$ and Robert Szipőcs ${ }^{1}$ \\ ${ }^{1}$ Wigner RCP, Inst. for Solid State Physics and Optics, P.O. Box 49, H-1525 Budapest, Hungary \\ ${ }^{2}$ Department of Dermatology, Semmelweis University, H-1085 Budapest, Hungary \\ ${ }^{3}$ PXE Reference Center (MAGEC Nord), Angers University Hospital, Angers, France
}

DOI: https://doi.org/10.14232/kvantumelektronika.9.8

\section{Introduction}

Pseudoxanthoma elasticum (PXE, OMIM\#264800) is an autosomal recessive metabolic disorder characterized by progressive ectopic mineralization of soft connective tissue [1]. To date, diagnosis of PXE is confirmed with skin biopsy and/or genotyping. Histopathology findings include fragmented, clumped and mineralized elastic fibers, and calcium deposits in the mid-dermis, mainly consisting of calcium hydroxyapatite and calcium hydrogen phosphate [2]. According to ultrastructural studies, two types of calcification were described: fine precipitates inside the elastic fibers in their center and bulky deposits which by surrounding the fibers, lead to deformation and breakage [3]. Coiled, split collagen fibers with irregular diameter and flower-like collagen fibrils are also observed sometimes [4].

Nonlinear microscopy (NLM) is a high-spatial resolution imaging technique that has been increasingly used in dermatological research including non-invasive detection of skin cancer [5] and stain-free examination of dermal connective tissue alterations [6-8]. Among the different NLM modalities, two-photon excitation fluorescence (TPEF) is suitable for the visualization of endogenous fluorophores, such as elastin and keratin, whereas chiral structure collagen generates second-harmonic generation (SHG) signal [9].

Recently, we have shown that NLM is able to visualize the histopathological alterations of the middermis in PXE-affected skin using formalin fixed, paraffin embedded (FFPE) and then deparaffinized PXE sections. Besides SHG imaging of collagen, we successfully visualized calcification and detrimental changes to elastic fibers with TPEF [10]. However, in this preliminary study, we captured two-channel NLM images (SHG and TPEF), where TPEF signals originating from elastin and calcification were detected by a single NDD channel. Thus, their optical differentiation was not feasible and solely was based on their morphology.

This year we have investigated fluorescent properties of these two tissue components and found that in fresh-frozen PXE sections, they have a broad, overlapping emission spectrum [11,12]. Introducing a normalized 3D color vector representation of their emission spectra, we have learned that distinction of elastin and calcification for high contrast imaging is rather difficult (no data shown here). However, we realized that by applying some low concentration, (hence low absorption) eosin staining during the deparrafinization process of PXE sections, their spectral distribution considerably differs from each other, since eosin, as a pink (fluorescent) staining for basic tissue components has a relatively higher concentration in calcification. As a consequence, these two tissue components can be optically distinguished by laser scanning NLM, even without damaging the tissue due to laser light absorption in stained sections. 
In this work our aim was to develop an improved imaging and mathematical apparatus (spectral decomposition) to obtain three-color mosaic imaging of deparaffinized PXE sections using NLM, where elastin, calcification and collagen is visualized with distinct colors, to make images suitable for quantitative analysis and further investigation. By applying an artificial red, green, and blue color encoding for enhanced mapping co-localization of mineralization and elastic fibers, high spatial resolution, high contrast histological mosaic images have been recorded for low concentration eosin stained, deparrafinized PXE sections.

\section{Experimental design}

\subsection{Sample preparation}

Skin biopsy samples from PXE-affected areas of confirmed PXE patients were formalin-fixed and paraffin-embedded. For the NLM imaging, $20 \mu \mathrm{m}$ thick, deparaffinized, sections were prepared. For deparrafinization alcohol was used, in which a low concentration eosin stain was solved. As a result, the deparrafinized PXE sections had a light-pink colour in contrast to standard (white colour) deparrafinized PXE sections. For conventional histopathology, sections from the same skin biopsies were stained with Weigert's elastic (WE) and von Kossa (VK) stains. Histopathologic evaluation was performed by an expert dermatopathologist. PXE patients were diagnosed and managed at the PXE Reference Center (MAGEC Nord), Angers University Hospital, Angers, France, (protocol identifier: NCT01446380). Our present study was approved by the local Ethics Committee in Budapest, Hungary (SE TUKEB no. 193/2017).

\subsection{Nonlinear microscope imaging}

NLM imaging was carried out with a $\sim 20 \mathrm{MHz}$ repetition rate, sub-ps Ti:sapphire laser (FemtoRose 300 TUN LC, R\&D Ultrafast Lasers Ltd., Budapest, Hungary, (R\&D Ltd.)) operating at $800 \mathrm{~nm}$. To capture images, an Axio Examiner LSM 7 MP laser scanning two photon microscope system (Carl Zeiss, Germany) with modified detection optics was used with a 20x water immersion objective (W-Plan - APOCHROMAT 20x/1.0 DIC (UV) VIS-IR, Carl Zeiss, Germany). Individual image size was $420 \times 420 \mu \mathrm{m}^{2}$. Further details about the imaging setup can be found in Ref. [7]. TPEF and SHG signals were collected by two NDD detectors and visualized by the ZEN 3.0 SR software (Carl Zeiss AG, Germany). 460/50 nm (cyan) and 590/45 nm (orange) bandpass filters were used to capture TPEF signals of elastin and calcification, respectively, whereas for SHG, a 405/20 nm (violet, displayed as magenta) bandpass filter was used. In order to compare the results with our previous work, we used a 525/500 nm (green) filter as well. Mosaic images with an individual image size of $420 \times 420 \mu \mathrm{m}^{2}$ were obtained with the use of a custom-made stepping motor system (Mosaic v1.0, R\&D Ltd.). The acquired TPEF and SHG images were processed and assembled into two- or three-color mosaic images with ImageJ v1.46 software (NIH, Bethesda, MD, USA).

\subsection{Three-channel decomposition and inverse matrix calculation}

Overlapping emission spectra result in correlated detected signals, where signal intensities appearing in different detection channels are proportional to concentration of a given tissue component [13,14]. Three-channel decomposition has been reported in SRS microscopy [15]. During our work, we have developed a similar algorithm for decomposition of mixed TPEF and SHG signals and deduced contribution of each excited substance.

Briefly, first we acquired TPEF signals with cyan and orange emission filters and SHG signal with a viola filter from manually selected $132 \times 132 \mu \mathrm{m}^{2}$ regions with the highest SHG (collagen), $\mathrm{TPEF}_{\text {cyan }}$ (elastin) and $\mathrm{TPEF}_{\text {orange }}$ (calcium deposits) signal intensities, respectively $\left(\mathrm{I}_{405}, \mathrm{I}_{460}, \mathrm{I}_{590}\right)$. 
Selected regions correspond to areas where concentration of collagen, elastin and calcium deposit appears the highest, respectively. $\mathrm{I}_{405}, \mathrm{I}_{460}, \mathrm{I}_{590}$ are linear combinations of light emission from tissue components described by their concentrations (collagen $\left(\mathrm{C}_{\mathrm{Co}}\right)$, elastin $\left(\mathrm{C}_{\mathrm{E}}\right)$ and calcium deposits $\left(\mathrm{C}_{\mathrm{Ca}}\right)$ ). Contribution of each tissue component to measured SHG and TPEF intensities is described by matrix coefficients $a_{i, j}$ as listed in Eq. 1 . In each region, a $3 \times 3$ pixel area with the highest intensity value pixel was identified. Average $\mathrm{I}_{405}, \mathrm{I}_{460}, \mathrm{I}_{590}$ signal intensities were measured within these three areas, respectively. Measured average intensities were then normalized to their maximum, respectively. These computed intensity ratios were then used as matrix coefficients in transfer matrix [A] (Eq.1).

$$
\left[\begin{array}{l}
I 405(x, y) \\
I 460(x, y) \\
I 590(x, y)
\end{array}\right]=\left[\begin{array}{lll}
a 405, C o & a 405, E & a 405, C a \\
a 460, C o & a 460, E & a 460, C a \\
a 590, C o & a 590, E & a 590, C a
\end{array}\right]\left[\begin{array}{c}
C C o(x, y) \\
C_{E(x, y)} \\
C C a(x, y)
\end{array}\right]
$$

In vector form, measured fluorescence intensity $\underline{I}$ can be calculated as concentration vector $\underline{C}$ multiplied by transfer matrix [A], i.e. $\underline{I}=\underline{A} \underline{A}$. From this, inverse matrix of [A] can be defined. After calculation of matrix elements of $[\mathrm{A}]^{-1}$, relative concentrations of tissue components (at each pixel) can be deduced such as $\underline{C}=\underline{A}^{-1} \underline{I}$. Having matrix elements of $[\mathrm{A}]^{-1}$ calculated, image postprocessing has been applied to each pixel $(\mathrm{x}, \mathrm{y})$ of our three-channel images $\underline{I}^{(}(x, y)$ in order to get concentration maps $\underline{C}_{(x, y)}$. Decomposed single channel concentration maps have been merged and assembled into three-color mosaic images.

\section{Results}

\subsection{Histopathology}

Mid-dermis of PXE patient showed remarkable changes. WE staining displayed irregular, fragmented, thickened elastic fibers along with mineralized elastin (no Figure shown). VK staining exhibited mineral deposition and calcified elastic fiber clumps in mid-dermis. Disrupted, coiled collagen fibers around calcium deposits are also revealed. Papillary dermis and deep reticular dermis were unaffected in all patients.

\subsection{NLM imaging and three-channel decomposition}

In frozen and deparrafinized PXE sections, we found that emission spectra of elastin and calcification can hardly be distinguished in a four colour (viola/cyan/green/orange) vector space, however, we found that by using a low concentration eosin solution for deparrafinization these two tissue components can be optically distinguished by their fluorescence spectra: elastin exhibits a relatively higher TPEF signal in the cyan, whereas calcium deposits have relatively high TPEF signal in the orange detection channel. Reference images for determination of inverse matrix coefficients are shown in Figure 1. Linear combination of signal intensities detected in a single channel was described as discussed in Section 2.3. For simplicity, $\mathrm{C}_{\mathrm{Co}}, \mathrm{C}_{\mathrm{E}}$ and $\mathrm{C}_{\mathrm{Ca}}$ in Eq. 1 were set equal to 1. To exemplify, by substituting calculated coefficients we obtained transfer matrix [A] for PXE sample of Patient1 (Eq.2.), and then derived inverse matrix [A $]^{-1}$ (Eq.3).

$$
\left[\begin{array}{l}
I 405(x, y) \\
I_{460(x, y)} \\
I_{590(x, y)}
\end{array}\right]=\left[\begin{array}{ccc}
1 & 0.134 & 0.116 \\
0.056 & 1 & 0.356 \\
0.038 & 0.488 & 1
\end{array}\right]\left[\begin{array}{l}
1 \\
1 \\
1
\end{array}\right]
$$




$$
\left[\begin{array}{c}
C_{C o}(x, y) \\
C_{E}(x, y) \\
C_{C a(x, y)}
\end{array}\right]=\left[\begin{array}{ccc}
1.008 & -0.089 & -0.094 \\
-0.051 & 1.243 & -0.484 \\
-0.014 & -0.670 & 1.376
\end{array}\right]\left[\begin{array}{c}
I_{405(x, y)} \\
I_{460(x, y)} \\
I_{590(x, y)}
\end{array}\right]
$$

As the SHG signal of collagen is measured with a narrowband bandpass filter, both elastin and calcification TPEF results in a minimal contribution to SHG signal. However, collagen does exhibit low TPEF signal that appears in the cyan and orange channels. Calcification and elastin has wide, overlapping TPEF spectra that manifests in relatively high coefficients in both cyan and orange channels. Executing image post-processing using the retrieved inverse matrix resulted in spectrally

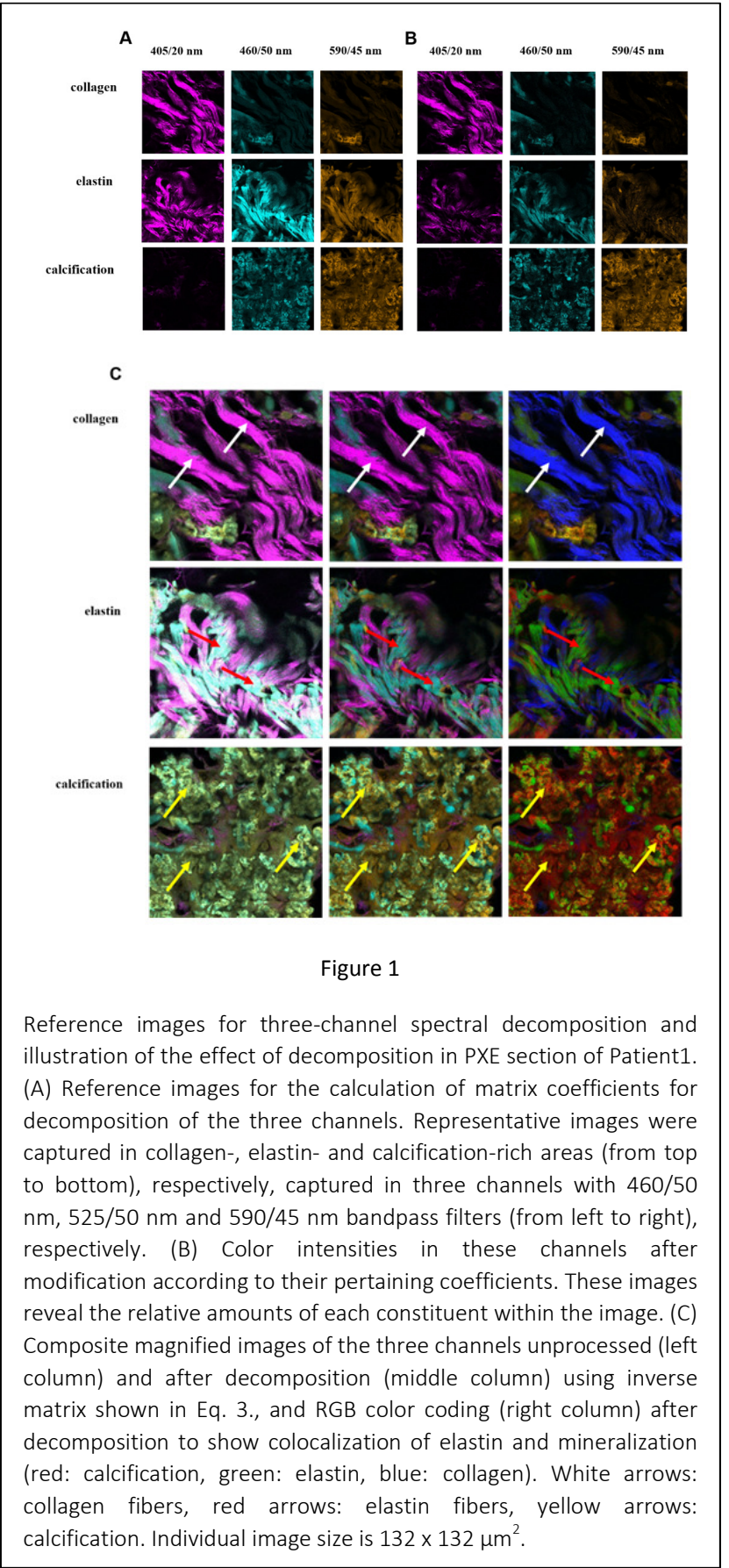
decomposed images, where crosstalk arising from the other components is considerably reduced and hence optical signals originating from each tissue component are well separated. Figure 1C displays composite of reference images before and after decomposition.

Applying the same calculations, we assembled mosaic of composite images (Figure 2). The effect of decomposition is demonstrated in Figure 2A (original) and Figure 2B (decomposed) for three-channel mosaic image of PXE section of Patient1. As we can see, decomposition of this mosaic image enabled to separate of collagen and elastic fibers. The contrast between the TPEF signal of elastin and calcification is considerably improved and areas, which are affected by calcification, are clearly circumscribed. The papillary dermis was not affected, similarly to histopathology. Elastic fibers in the mid-dermis are fragmented and polymorphous, as shown by the $\mathrm{TPEF}_{\text {cyan }}$ channel. Calcium deposits, revealed by the $\mathrm{TPEF}_{\text {orange }}$ channel occupy large areas in the mid-dermis and dislocate clumped elastic fibers. In order to demonstrate the improved chemical selectivity of our presented method, two color composite mosaic image applying the SHG and green TPEF detection channels is shown in Figure $2 \mathrm{C}$. In order to visualize calcified elastic fibers, we applied artificial red, green and blue colors to display calcification, elastin and collagen, respectively, after decomposition (Figure 2D). Colocalization is revealed by addition of red and green colors appearing in yellow color in Fig. 2D. 

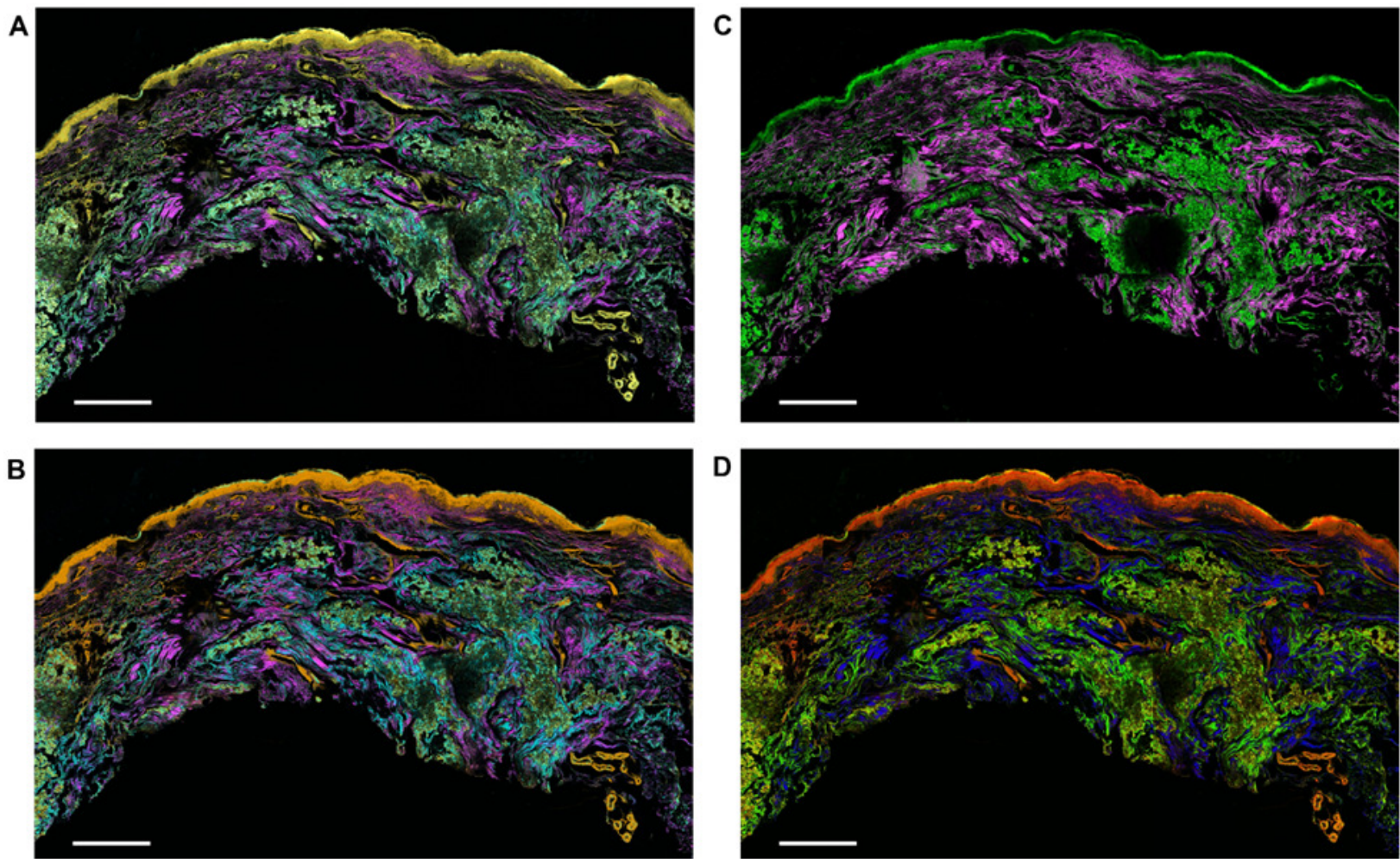

Figure 2

Composite of two-photon excitation fluorescence (TPEF) and second-harmonic generation (SHG) mosaic images of PXE-affected skin samples Excitation wavelength is $800 \mathrm{~nm}$. For images (A,B), TPEF signal is captured by using two different bandpass filters. Cyan (460/50 nm) and orange $(590 / 45 \mathrm{~nm})$ emission filters were chosen to detect high portion of TPEF signals originating from elastin and calcification, respectively, with maximum contrast for spectral decomposition. For mosaic image (C), TPEF signal of elastin and calcification together was collected by a single green (525/50 $\mathrm{nm}$ ) bandpass filter. SHG signal of collagen was spectrally selected by a narrowband 405/20 nm filter for (A,B,C) images and displayed by magenta color. Note that keratin has a broad TPEF signal appearing in the green, orange, and to some extent, in cyan channel. Individual images of $420 \times 420$ $\mu \mathrm{m}^{2}$ were assembled into (A,B,C,D) mosaic images of PXE-affected skin of Patient1. (A) Three-channel detection, composite mosaic image of TPEF cyan, TPEF orange and SHG signals. (B) Three-channel composite of TPEF cyan, TPEF orange and SHG mosaic images after spectral decomposition using the inverse matrix described in Eq.3. (C) Two-channel detection, composite mosaic image of SHG and green TPEF channel. (D) Artificial red, green and bue colored images (red: calcification, green: elastin, blue: collagen), colocalization of elastin and calcification appears in yellow color.

\section{Discussion}

Histological alterations in PXE can be observed not just in affected areas, but also in clinically healthy appearing skin, and also in patients withouth characteristic skin signs dermis [16]. As subtle skin changes seen in histopathology of PXE often precede organ damage, the assessment of skin status should be of primary importance.

In our present paper, we examined TPEF signals of eosin solution stained elastin and calcium deposits and SHG signal of collagen in deparrafinized PXE sections by applying different bandpass filters. Using an inverse matrix-based decomposition method, separation of the overlapping emitted signals of elastin, calcification and collagen, and precise calculation of spatial distribution of these tissue components was possible only after an eosin solution staining process. After executing decomposition, we were able to distinguish intact and calcified elastic fibers, as well as bulky calcification and clumps of fragmented elastic fibers from each other. Besides, we also showed where these colocalize within the sample. Our data suggests that our presented decomposition method has important advantageous features compared to conventional histopathology since our decomposed NLM images could be directly used for quantitative analysis using computers. Furthermore, fine structures can be better identified due high selectivity and high spatial resolution. 


\section{Acknowledgements}

This research was funded by the National Research, Development and Innovation Fund of Hungary contract No. K_129047 of Wigner RCP and FK_131916 of Semmelweis University. Skin samples were provided by PXE Reference Center (MAGEC Nord), Angers University Hospital, Angers, France in the framework of COST Action No. CA16115. The authors thank Enikö Kuroli for the histopathological assessment of the skin sections.

\section{References}

[1] J. Uitto, Q. Jiang, A. Varadi, L.G. Bercovitch, S.F. Terry, Expert Opin Orphan Drugs 2, 567 (2014) https://doi.org/10.1517/21678707.2014.908702

[2] M.J. Hosen, A. Lamoen, A. De Paepe, O.M. Vanakker, , Scientifica (Cairo), 598262 (2012) https://doi.org/10.6064/2012/598262

[3] B. Marconi, I. Bobyr, A. Campanati, E. Molinelli, V. Consales, V.Brisigotti, M. Scarpelli, S. Racchini, A. Offidani, Intractable Rare Dis Res 4,113 (2015) https://doi.org/10.5582/irdr.2015.01014

[4] D.P. Germain, Orphanet J. Rare Dis. 12, 85 (2017)

https://doi.org/10.1186/s13023-017-0639-8

[5] E. Dimitrow, M. Ziemer, M.J. Koehler, J. Norgauer, K. Konig, P. E M. Kaatz, J. Invest. Dermatol. 129, 1752 (2009) https://doi.org/10.1038/jid.2008.439

[6] K. Lorincz, D. Haluszka, N. Kiss, N. Gyongyosi, A. Banvolgyi, R. Szipocs, N.M. Wikonkal, Arch. Dermatol. Res. 309, 209 (2017)

https://doi.org/10.1007/s00403-017-1715-6

[7] D. Haluszka, K. Lorincz, N. Kiss, R. Szipocs, E. Kuroli, N. Gyongyosi, N.M. Wikonkal, Biomed Opt Express 7, 4480 (2016)

https://doi.org/10.1364/BOE.7.004480

[8] N. Kiss, D. Haluszka, K. Lőrincz, E. Kuroli, J. Hársing, B. Mayer, S. Kárpáti, G. Fekete, R. Szipőcs, N. Wikonkál, M. Medvecz, Arch. Dermatol. Res. 310, 463 (2018) https://doi.org/10.1007/s00403-018-1835-7

[9] S.W. Perry, R.M. Burke, E.B. Brown, Ann. Biomed. Eng. 40, 277 (2012) https://doi.org/10.1007/s10439-012-0512-9

[10] N. Kiss, L. Fesus, S. Bozsanyi, F. Szeri, M. Van Gils, V. Szabo, A.I. Nagy, B. Hidvegi, R. Szipocs, L. Martin, O. Vanakker, T. Aranyi, B. Merkely, N.M. Wikonkal, M. Medvecz, Lasers Med. Sci. 35, 1821-1830 (2020) https://doi.org/10.1007/s10103-020-03027-w

[11] M. Fitzmaurice, J.O. Bordagaray, G.L. Engelmann, R. Richards0Cortum, T. Kolubayev, M.S. Feld, N.B. Ratliff, J.R. Kramer, Am. Heart J. 118, 1028 (1989)

https://doi.org/10.1016/0002-8703(89)90239-1

[12] L.M. Baugh, Z. Liu, K.P. Quinn, S. Osseiran, C.L. Evans, G.S. Huggins, P.W. Hinds, L.D. Black, I. Georgakoudi, Nat Biomed Eng 1, 914 (2017)

https://doi.org/10.1038/s41551-017-0152-3 
[13] P.P. Provenzano, K.W. Eliceiri, L. Yan, A. Ada-Nguema, M.W. Conklin, D.R. Inman, P.J. Keely, Microscopy and microanalysis 14, 532 (2008)

https://doi.org/10.1017/S1431927608080884

[14] R. Carriles, D.N. Schafer, K.E. Sheetz, J.J. Field, R. Cisek, V. Barzda, A.W. Sylvester, J.A. Squier, , The Review of Scientific Instruments 80, 081101 (2009)

https://doi.org/10.1063/1.3184828

[15] L. Shi, C. Zheng, Y. Shen, Z. Chen, E.S. Silveira, L. Zhang, M. Wei, C. Liu, C. de SenaTomas, K. Targoff, W. Min, Nat Commun 9, 2995 (2018)

https://doi.org/10.1038/s41467-018-05401-3

[16] N. Chassaing, L. Martin, P. Calvas, M. Le Bert, A. Hovnanian, J. Med. Genet. 42, 881 (2005) https://doi.org/10.1136/jmg.2004.030171 